\title{
Load-aware Transparent Reach Maximization in Flexible Optical Networks
}

\author{
Alberto Bononi, Paolo Serena, Giorgio Picchi \\ Dip. Ing. Informazione, Università di Parma, Parma, Italy. \\ Email: alberto.bononi@unipr.it
}

\author{
Annalisa Morea \\ Alcatel-Lucent Bell Labs France, Nozay, France. \\ Email: annalisa.morea@alcatel-lucent.com
}

\begin{abstract}
We propose a novel load-aware powerselection strategy for maximizing the transparent reach in dispersion-uncompensated flexible optical networks with coherent modulation formats, and we quantify the savings in opto-electronic regenerations with respect to using the standard full-load transparent reach.
\end{abstract}

\section{INTRODUCTION}

We consider the transmission of wavelength division multiplexed (WDM) dual-polarization (DP) optical digital signals with coherent detection through a dispersion uncompensated (DU) flexible optical network [1][3], i.e., a circuit-switched WDM fiber-optic network where each fiber carries at most $W$ wavelengths with possibly mixed modulation formats and where each alloptical segment of a circuit (i.e., a lightpath) occupies a wavelength. From the source, the destination may be transparently reached via a single lightpath (i.e., without opto-electronic regeneration (OER) or through a concatenation of lightpaths on (possibly) different wavelengths, with OER from one lightpath to the next one. All-optical wavelength conversion at a node is excluded in this analysis. To minimize the number of costly OER, the quality-of-transmission (QoT) aware routing and wavelength assignment (RWA) algorithm first tries to set-up a circuit along a single lightpath. Such a connection may not be feasible for two reasons:

i) unavailability of the same wavelength across successive fibers along the lightpath, leading to wavelength blocking (WB);

ii) the received signal to noise ratio (SNR) for the considered modulation format is below a required minimum value $S_{0}$, leading to $S N R$ blocking (SB).

In this paper we concentrate on physical-layer design issues connected with RWA, and in particular on SB due to accumulation of nonlinear optical impairments along lightpaths. A common approach to resolve SB is to accept from the RWA only lightpaths whose physical length is below a threshold, which we call the fullload $(F L)$ reach [1], [3]. The FL reach is the maximum lightpath length guaranteeing a received SNR above $S_{0}$ when all $W$ wavelengths on all lightpath fibers are occupied. The FL reach is used regardless of the actual wavelength load $u$, i.e., the fraction of wavelengths actually utilized by lightpaths in the network. An optimal distance-independent power selection policy for signaling on the new lightpath has recently been proposed in [3], based on the incoherent Gaussian Noise (GN) model [4].

Using the FL reach is clearly conservative, since wavelengths saturation at the network core prevents the average network load $u$ to reach unity. Depending on network size and connectivity, such a load may even be less than 0.5. In this paper, therefore, we propose the use of a new load-dependent reach and a corresponding optimal power, and quantify the potential OER savings with respect to the approach in [3].

\section{NONLINEAR TRANSMISSION MODEL WITH ON/OFF RANDOM TRAFFIC}

We study the transparent transmission of a DP signal across a selected lightpath from source to destination. A lightpath is a sequence of $H$ hops across access nodes (i.e., nodes where circuits may originate and terminate), where the k-th hop is a concatenation of $\mathcal{S}_{k}$ amplified spans followed by the crossing of the $\mathrm{k}$-th intermediate node, for $k=1, \ldots, H$. A span is composed of a transmission fiber followed by an endline lumped optical amplifier. A node is composed of a wavelength demultiplexer, add/drop block and output multiplexer. In our calculations, the losses in crossing a node will be equivalent to those in crossing one span. Our reference lightpath is composed of $N_{s}=\sum_{k=1}^{H} \mathcal{S}_{k}$ identical spans. We assume that each of the $W-1$ remaining wavelengths of the k-th hop independently carries an interferer lightpath (hence carries power) with known probability $u_{k}, k=1, \ldots, H$. Within a firstorder perturbation analysis, the received SNR over the bandwidth of the DP signal of interest after propagation across the reference lightpath is [7], [8]:

$$
S N R\left(P, N_{s}, \mathbf{u}\right)=\frac{P}{\beta\left(N_{s}+H\right)+a_{N L}\left(N_{s}, \mathbf{u}\right) P^{3}}
$$

where $P$ is the DP reference signal power at the input of each transmission fiber section; $N_{A} \triangleq \beta\left(N_{s}+H\right)$ is 
the amplified spontaneous emission (ASE) power from the $\left(N_{s}+H\right)$ optical amplifiers, with $\beta \triangleq h \nu F G B_{r x}$, where $h$ is Planck's constant, $\nu$ is the optical carrier frequency, $F$ is the amplifier noise figure, $G$ is the amplifier gain (equal to the span loss) and $B_{r x}$ is the receiver equivalent noise bandwidth; $a_{N L}$ is the nonlinear interference (NLI) coefficient [4], [7] which depends on the number of spans and on the wavelength load vector $\mathbf{u}=\left[u_{1}, \ldots, u_{H}\right]$, which in turn depends on the offered traffic and on the RWA algorithm. Since the number of interfering wavelengths is a random variable (RV), then also the $a_{N L}$ coefficient and the received SNR are $\mathrm{RVs}$, whose statistics depend on $\mathbf{u}$. SNR is deterministic only in the two limiting cases ${ }^{1} \mathbf{u}=\mathbf{0}$ (single channel operation) and $\mathbf{u}=\mathbf{1}$ (full load operation) where there is no variability of the number of interfering lightpaths.

We assume the digital signal is coded with a forward error-correction code whose SNR threshold for the signal modulation format is $S_{0}$. We declare an SB event when $S N R\left(P, N_{s}, \mathbf{u}\right)<S_{0}$. The circuit is in that case routed via multiple lightpaths and requires OER at intermediate nodes.

\section{SUMMARY OF RESULTS}

We anticipate in this section our main results regarding the load-dependent reach. The design of point-topoint DU transmission systems for high symbol rate DP WDM coherent systems is based on the received SNR contours versus transmitted power ${ }^{2} P$ and number of spans $N_{s}$ [9]. In a networking scenario, however, the SNR is a RV. We thus propose to base the transmission design of DU networks on contours of the SNR blocking probability

$$
P_{S B} \triangleq \operatorname{Pr}\left\{S N R\left(P, N_{s}, \mathbf{u}\right)<S_{0}\right\}
$$

at fixed load $\mathbf{u}$ versus both power per channel $P$ and number of spans $N_{s}$. The QoT-aware RWA, which has only knowledge of the load vector $\mathbf{u}$, can safely declare that a new lightpath of length $N_{s}$ will have sufficient SNR at destination if $P_{S B}$ is less than or equal to a target level ${ }^{3} \mathcal{P}_{S B}$ for the selected lightpath modulation format. From the $P_{S B}$ contours at the target level we can visualize both the maximum number of spans that can be bridged without OER (i.e., the load-dependent reach) and the associated optimal power.

For simplicity, in the numerical calculations in this paper we assumed the load $u_{k}$ and the spans per hop $\mathcal{S}_{k}$ are uniform at all hops $k=1, \ldots, H$ and all signals

\footnotetext{
${ }^{1}$ here boldface $\mathbf{0}$ is the vector of all zeros, and boldface $\mathbf{1}$ the vector of all ones.

${ }^{2} \mathrm{We}$ assume here all signals in the network have the same power.

${ }^{3}$ Of course, there is a chance that the RWA is wrong, in which case the receiver signals back to the controller and the lightpath is torn down and segmented into more lightpaths.
}

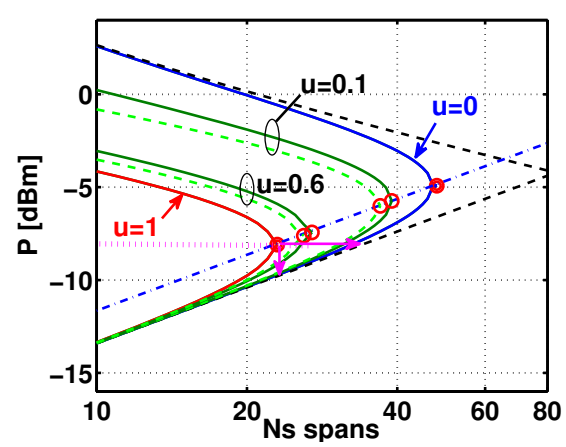

Fig. 1. Horizontal cuts (contours) of SNR-blocking (SB) probability at level $\mathcal{P}_{S B}$ versus power $P$ and number of spans $N_{S}$ at load values $u=[0,0.1,0.6,1]$. All pairs $\left(P, N_{s}\right)$ inside each contour yield $\operatorname{Pr}\left\{S N R\left(P, N_{s}, \mathbf{u}\right)<S_{0}\right\} \leq \mathcal{P}_{S B}$. The target SNR is here $S_{0}=$ $9.8 \mathrm{~dB}$, i.e., bit error rate $>10^{-3}$ for a DP-QPSK format. For $u=$ 0.1 and $u=0.6$ the dashed green contours are at level $\mathcal{P}_{S B}=$ $10^{-3}$ and the solid green contours at level $\mathcal{P}_{S B}=0.5$. Linear and nonlinear asymptotes shown as black dashed lines. Locus of maximum reach points shown as blue dashed-dotted line parallel to (lower) linear asymptote, upward shifted by $1.76 \mathrm{~dB}$. Link data: $100 \mathrm{~km}$ fiber spans with dispersion $D=2 \mathrm{ps} / \mathrm{nm} / \mathrm{km}$, attenuation $\alpha=0.2 \mathrm{~dB} / \mathrm{km}, n_{2}=$ $2.5 \cdot 10^{-20} \mathrm{~m}^{2} / W, A_{\text {ef } f}=80 \mu \mathrm{m}^{2}$ at $\lambda=1550 \mathrm{~nm}$. Amplifiers noise figure $F=4 \mathrm{~dB} . W=81$ wavelengths (reference is center channel. Symbol rate $R=10 \mathrm{Gbaud}$ per channel. Spacing $\Delta f=12.5 \mathrm{GHz}$ (spectral fill factor $\eta=\frac{R}{\Delta f}=0.8$ ). $\mathcal{S}=2$ span/hop.

have the same power and modulation format, although the theory is developed for non-uniform $u_{k}, \mathcal{S}_{k}$, and can be straightforwardly extended to mixed modulation formats. Fig. 1 shows the SB probability contours for a WDM DP quadrature phase shift keying (DP-QPSK) system with $W=81$ wavelengths and $R=10$ Gbaud signals transmitted over $N_{s} 100 \mathrm{~km}$ DU spans of nonzero dispersion fiber (NZDF). The points of maximum reach are marked by red circles in the figure. We indicate their coordinates as $\left[N_{0}(u), P_{0}(u)\right]$. Rectangular signal spectra were assumed in the $a_{N L}$ calculations according to a recent extension [10] of the coherent GN model [4]. SB probability calculations will be detailed in Section IV.

At $u=1$ and $u=0$ the SB contours at all $\mathcal{P}_{S B}$ levels coincide. For all $\left(P, N_{s}\right)$ pairs inside the region delimited by the upper branch $P_{M}$ and the lower branch $P_{m}$ of the contours (red contour at $u=1$ and blue contour at $u=0)$ the SB probability is exactly zero, while outside it is exactly 1 . Instead, at any other intermediate load $0<u<1$ the contours vary with the value of $\mathcal{P}_{S B}$. For instance, at loads $u=0.6$ and $u=0.1$ the dashed green lines show the contours at level $\mathcal{P}_{S B}=10^{-3}$, while the dark green solid lines those at level $\mathcal{P}_{S B}=0.5$. We note that the dashed green and the dark solid green contours are quite close to each other (they get closer and closer as the number of spans per hop $\mathcal{S}$ decreases). This is an indication that the transition of SB probability from 
zero to 1 is rather sharp, and SB contours at practical levels will be close to the one at level $\mathcal{P}_{S B}=0.5$, which - as we shall prove in Section IV - coincides with the following deterministic SNR contour at level $S_{0}$ :

$$
S N R\left(E\left[a_{N L}\right]\right) \triangleq \frac{P}{\beta\left(N_{s}+H\right)+E\left[a_{N L}\left(N_{s}, u\right)\right] P^{3}}=S_{0}
$$

where the traffic-averaged coefficient $E\left[a_{N L}\right]$ is used. For such a deterministic SNR contour we easily prove in Appendix 1 that its locus of maximum reach points, as $E\left[a_{N L}\right]$ varies, lays on the dashed-dotted straight line shown in Fig. 1 parallel to the (lower) linear asymptote and shifted by $10 \log (3 / 2) \cong 1.76 \mathrm{~dB}$ above that. In the $\log -\log$ plot of Fig. 1 the linear asymptote and hence the dashed-dotted line have slope $1 \mathrm{~dB} /$ decade, hence the magenta arrows in the figure indicate $1.76 \mathrm{~dB}$ on each axis direction.

This has a fundamental consequence, first noted in [3]. If we set $P$ to the full load value $P_{0}(1)$ (magenta dotted line in the figure) then the ratio between the fullload reach $N_{0}(1)$ and the maximum reach $N_{0}(u)$ at any other load $u<1$ is always smaller than $2 / 3$. Thus, if in the QoT-aware RWA algorithm we set the maximum non-regenerative reach to its full-load value $N_{0}(1)$, at most we under-estimate the true reach by a factor $1 / 3$, i.e., by 33\% [3]. This was the rationale for proposing a full-load QoT-aware RWA design using the distanceindependent full load power $P_{0}(1)$ in [3].

However, suppose for instance the actual load is only $u=0.1$. If we use the correct maximum reach power $P \equiv P_{0}(u)=-6 \mathrm{dBm}$ (see green dashed contour at $u=$ 0.1 ) we find that the actual reach is $N_{0}(u)=37$ spans, which compared with the full-load reach $N_{0}(1)=23$ spans gives a transparent reach under-estimation by the full-load RWA with respect to the true reach by

$$
\mathcal{U} \triangleq \frac{N_{0}(u)-N_{0}(1)}{N_{0}(u)} 100=37.8 \%
$$

which is above $33 \%$. This means that if we know the average wavelength load $u$ and then select the optimal maximum reach power $P_{0}(u)$ (whose explicit formula is provided later in eq. (26)), the reach under-estimation with respect to the true reach $N_{0}(u)$ when we use the full-load RWA can be larger than 33\%.

By solving the implicit equation (25) of Section IV we can directly obtain the maximum reach values $N_{0}(u)$ at any load $u$ and the corresponding optimal power $P_{0}(u)$.

Fig. 2(top) shows $N_{0}(u)$ and Fig. 2(bottom) shows under-estimation $\mathcal{U}$ in (4), both plotted versus transmission fiber dispersion $D$ at several load values. We note a diminishing under-estimation at both increasing dispersion and load. For standard single-mode fiber (SMF) with $D=17 \mathrm{ps} / \mathrm{nm} / \mathrm{km}$, and at a symbol rate $R=$ 10Gbaud, the under-estimation is below $33 \%$ at loads
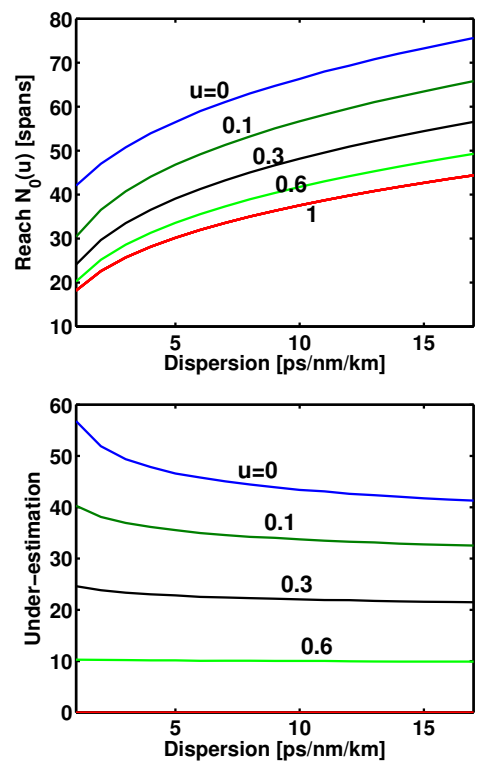

Fig. 2. (Top) maximum reach $N_{0}(u)$ of same SB probability contours at $\mathcal{P}_{S B}=10^{-3}$ as in Fig. 1, versus transmission fiber dispersion $D$ at several load values. (Bottom) Corresponding under-estimation (4). Data: $W=81, R=10 \mathrm{Gbaud}, \Delta f=12.5 \mathrm{GHz}, \mathcal{S}=2, z_{A}=$ $100 \mathrm{~km}, S_{0}=9.8 \mathrm{~dB}, F=4 \mathrm{~dB}, \mathcal{P}_{S B}=10^{-3}$.

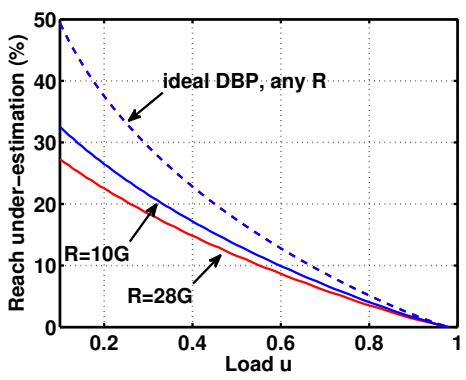

Fig. 3. Reach under-estimation versus load $u$ in a DU SMF link $(D=17 \mathrm{ps} / \mathrm{nm} / \mathrm{km})$ with $W=81$ WDM DP-QPSK channels at $R$ Gbaud/channel, at SNR blocking probability $\mathcal{P}_{S B}=10^{-3}$, with $z_{A}=100 \mathrm{~km}$ per span, $\mathcal{S}=2$ spans per hop, bandwidth efficiency $\eta=\frac{R}{\Delta f}=0.8$, and amplifiers noise figure $F=4 \mathrm{~dB}$.

$u \geq 0.1$. The under-estimation is also decreasing as we increase the channel symbol rate $R$ (recall that we also scale the frequency spacing $\Delta f=R / \eta$ ) and thus singlechannel nonlinear effects become dominant. However, when single-channel nonlinearity is fully compensated for by ideal digital-backpropagation (DBP), even at rates $R \geq 28 \mathrm{Gbaud}$ on SMF the picture goes back to the large gap between full load and single-channel contours, as in Fig. 1. Evidence here is provided by the reach underestimation versus load curves in Fig. 3, obtained at both $R=10 \mathrm{Gbaud}$ and $R=28 \mathrm{Gbaud}$ on SMF fiber, both without nonlinearity compensation and with ideal DBP. Note that with ideal DBP only cross-nonlinear effects 


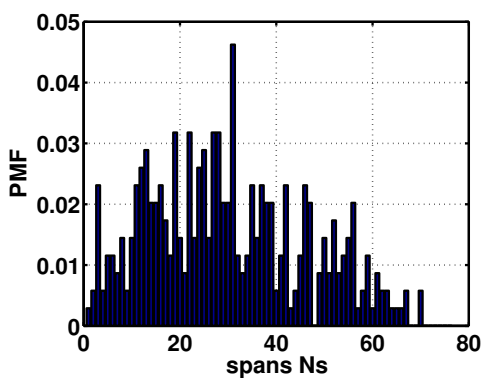

Fig. 4. Normalized histogram of lightpath lengths (spans) obtained from one path-setup simulation in a 46-node U.S. network at first wavelength blocking. Measured load was $u=0.46$, with a total of 346 lightpaths at WB.

remain, and the reach $N_{0}$ is essentially independent of the channel symbol rate and just depends on bandwidth efficiency $\eta$ [12].

We next need to quantify the savings in OER when using either the full-load RWA or our load-aware RWA. A quick approximate quantification is obtained as follows. We obtain the distribution of the lightpath length $N_{s}$ (spans) in the network from simulations when $S N R$ blocking is neglected. Each circuit is set up on a single lightpath until the first WB, when the measured load is $u$. Let the obtained normalized histogram of lightpath lengths $N_{s}$ (i.e., the empirical probability mass function (PMF) of $N_{s}$ ) be $P\left(N_{s}, u\right)$, which depends on both $N_{s}$ and load $u$. Fig. 4 shows an instance of such a PMF at first WB from one simulation in the 46-node U.S. network (US) [13] when the traffic matrix is uniform over the nodes and shortest path is selected. The average load was $u=0.46$. From the PMF, we can estimate the expected number of required OER when the reach is $N_{0}$ as

$$
E\left[\text { oer } \mid N_{0}\right]=\sum_{N_{s}=1}^{N_{\max }} P\left(N_{s}, u\right)\left(\left\lceil\frac{N_{s}}{N_{0}}\right\rceil-1\right)
$$

where $N_{\max }$ is the maximal $N_{s}$ in the network, and $\lceil x\rceil$ is the ceiling function. The percent savings $\mathcal{R}(u)$ in OER operations using our load-aware RWA with respect to the full-load RWA is

$$
\mathcal{R}(u)=\frac{E\left[\text { oer } \mid N_{0}(1)\right]-E\left[\text { oer } \mid N_{0}(u)\right]}{E\left[\text { oer } \mid N_{0}(1)\right]} \cdot 100 .
$$

Note that whenever $N_{0}(1)<N_{\max }<N_{0}(u)$ the savings are $100 \%$ since no regenerations are required with the load-aware RWA. Fig. 5 shows both the underestimation $\mathcal{U}$ eq. (4) and the savings $\mathcal{R}(u)$ eq. (6) versus load $u$ at WB for both the U.S. network and a 20-node European (EU) network [14] for a $\mathrm{W}=89$ wavelengths WDM DP-QPSK system at $R=28 \mathrm{Gbaud}$ and spacing $\Delta f=35 \mathrm{GHz}$ over SMF fiber $(D=17 \mathrm{ps} / \mathrm{nm} / \mathrm{km})$. The remaining parameters are as in Fig. 1. The results of 100

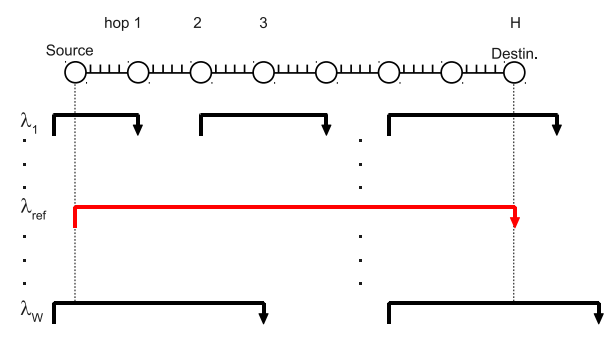

Fig. 6. Example of link spanned by reference lightpath at $\lambda_{\text {ref }}$ from Source to Destination node, with $H=7$ hops and and $\mathcal{S}=5$ spans per hop. Interfering lightpaths on wavelengths $\lambda_{1}$ through $\lambda_{W}$ may enter/exit at any node.

different runs are reported in the figures. Squares are for ideal DBP, crosses are without DBP. OER savings of more than $20 \%$ and $38 \%$ are obtained in the US and EU networks, respectively. Using single-channel nonlinearity suppression with an ideal DBP increases the savings to more than $50 \%$ in the US and $60 \%$ in the EU networks, respectively. The important message is that underestimation is not a good indicator of OER savings. Even at relatively small under-estimations as in the EU network, the savings in OER with respect to the standard full-load choice $N_{0}(1)$ can be significant. The reason is that $\mathcal{R}(u)$ depends both on the difference $N_{0}(u)-N_{0}(1)$ and on how quickly the PMF decreases to zero beyond $N_{0}(1)$.

\section{Details of Reach Analysis}

While the objective of the previous sections was to quantify the potential gain in maximum reach and OER savings of a load-aware RWA design, this section will provide all the analytical details necessary to explain the presented numerical results.

Fig. 6 sketches a link from source node $\mathrm{S}$ to destination node D for a reference lightpath at wavelength $\lambda_{\text {ref }}$. The number of hops is $H$ and generic hop $k$ is composed of $\mathcal{S}_{k}$ spans. Traffic on the reference lightpath enters at $\mathrm{S}$ and exits at D. On all other wavelengths, traffic may exit at an intermediate node, then re-enter at a later node (with different data), then exit, and so forth. Each thick line with ending arrow indicates a lightpath. We define

$u_{k}=\operatorname{Pr}\{$ a wavelength is carrying a lightpath at hop $k\}$.

This quantity can be estimated as the long-run average number of active lightpaths at hop $k$, divided by the total number of wavelengths $W$. The load vector $\mathbf{u}=\left[u_{1}, \ldots, u_{H}\right]$ is the only "traffic parameter", which depends on the QoT-aware RWA.

In DU networks, or more generally in networks with large-enough residual dispersion per span such that the coherent GN model [4] holds, the received SNR of the DP reference signal when all WDM interferers propagate 

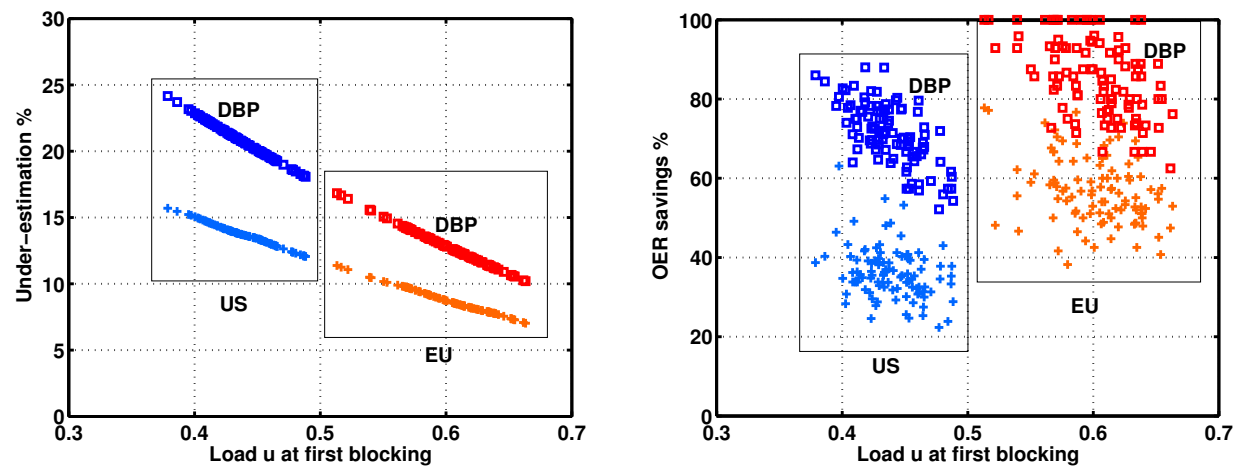

Fig. 5. (Left) Underestimation $\mathcal{U}$ eq. (4) and (Right) regenerations savings $\mathcal{R}$ eq. (6) vs load $u$ at first wavelength blocking in the U.S. and European (EU) networks for a W=89 wavelengths WDM DP-QPSK system at $R=28$ Gbaud and $\eta=0.8$ over SMF fiber $(D=17 \mathrm{ps} / \mathrm{nm} / \mathrm{km})$. Remaining parameters as in Fig. 1. Squares: DBP. Crosses: without DBP.

along the S-D path together with the reference signal is given by eq. (1), where the NLI power $P_{N L I}=a_{N L} P^{3}$ is [4]-[6], [10]:

$P_{N L I}=B_{r x} \frac{16}{27} \iint\left|\mathcal{K}\left(f_{1} f_{2}\right)\right|^{2} G\left(f_{1}\right) G\left(f_{2}\right) G\left(f_{1}+f_{2}\right) d f_{1} d f_{2}$

where $B_{r x}$ is the noise equivalent bandwidth of the receiver, $G(f)$ is the power spectral density (PSD) of the DP WDM comb, and $\mathcal{K}(v)$ is the (un-normalized) link kernel from node $\mathrm{S}$ at coordinate $z=0$ to node $\mathrm{D}$ at coordinate $z=L$, with $v=f_{1} \cdot f_{2}$, whose expression in absence of dispersion slope is [5], [6]

$$
\mathcal{K}(v)=\int_{0}^{L} \gamma(s) \mathcal{G}(s) \mathrm{e}^{-j \frac{(2 \pi)^{2} v}{2} C(s)} d s
$$

with $\gamma$ the nonlinear fiber coefficient, $\mathcal{G}(s)=$ $\exp \left(\int_{0}^{s} g(u) d u\right)$ the gain from 0 to $s \in[0, L]$, and $C(s)=-\int_{0}^{s} \beta_{2}(u) d u$ the cumulated dispersion from 0 to $s$. In (8), $v$ plays the role of a squared frequency. We assume that link gain/loss and total dispersion are perfectly compensated for at the coherent receiver.

Assuming channels have a rectangular spectrum, Fig. 7 shows as colored "islands" the integration domain of the double frequency integral in (7) [4], [10]. If any of the WDM signals is switched off, we have to switch off its corresponding PSD and thus remove the corresponding islands (e.g. when removing the second last WDM channel, those islands hatched in cyan in the figure). Fig. 7 shows one contour level of the function $\left|\mathcal{K}\left(f_{1} f_{2}\right)\right|^{2}$. Such contours are hyperbolas, as they just depend on the product $v=f_{1} \cdot f_{2}$. The central island accounts for the single channel interference (SCI). The remaining islands along the axes account for cross-channel interference (XCI), which also includes polarization-dependent effects. Off-axes islands account for four-wave mixing (FWM) among channels. FWM is normally negligible in DU links [4]. Neglecting FWM, the NLI coefficient

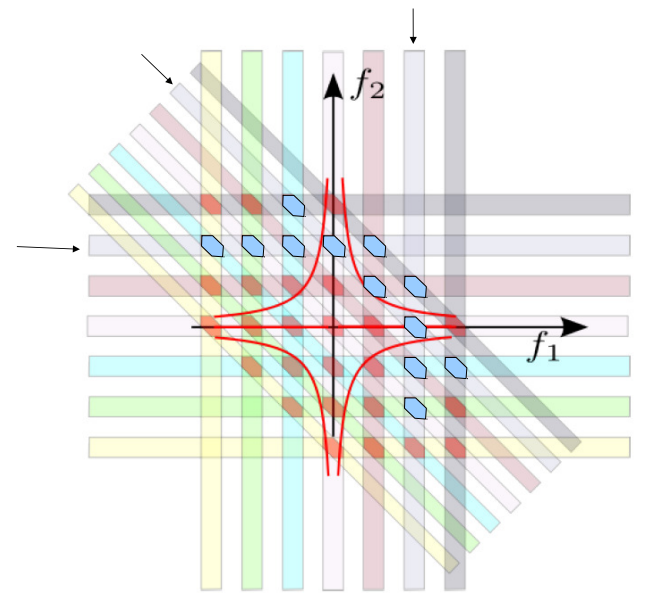

Fig. 7. Example of double integration domain (red and cyan "islands") in eq. (7) when spectra are rectangular with uniform channel spacing. Vertical stripes correspond to $G\left(f_{1}\right)$, horizontal stripes to $G\left(f_{2}\right)$, and 45-degrees tilted stripes to $G\left(f_{1}+f_{2}\right)$. When switching a channel OFF (e.g. the second last WDM channel) the corresponding PSD is zeroed, and so are the corresponding islands (those marked in cyan in the example).

can be written as

$$
a_{N L}=a_{S C I}+a_{X C I}
$$

and in [10] we found that with rectangular spectra the SCI coefficient in the coherent GN model may be well approximated at large enough symbol rate/dispersion as:

$a_{S C I} \cong \frac{16}{27} \frac{B_{r x}}{B_{0}}\left[\frac{4}{B_{0}^{2}} \int_{0}^{\left(B_{0} / 2\right)^{2}}|\mathcal{K}(v)|^{2} \log \left(\frac{\left(B_{0} / 2\right)^{2}}{v}\right) d v\right]$

where $B_{0}$ is the reference signal one-sided equivalent nonlinear optical bandwidth. It corresponds to integration of (7) over a square of edge $B_{0}$ centered at the origin [4]. In the numerical calculations in Section III we assumed $B_{0} \equiv k_{N L} R$ and $B_{r x}=k_{L} R$ where $k_{L}$ is 
found by bit-error rate (BER) matching with simulations in the linear regime, and once $k_{L}$ is fixed, then $k_{N L}$ is found by matching with simulated SNR contours. The values we found to best match our simulations are $k_{L}=1$ (i.e., ideal matched filtering - a normal situation in presence of a linear equalizer) and $k_{N L}=1.25$.

When all pumps propagate together with the signal, by generalizing the coherent $\mathrm{GN}$ model calculations with rectangular signal spectra in [10] we find that the dualpolarization XCI NLI coefficient can be approximated at large-enough channel spacing times symbol rate product $\Delta f \cdot R$ as :

$a_{X C I} \cong \frac{16}{27} \frac{B_{r x}}{B_{0}}\left[\sum_{p \neq 0} \frac{4}{B_{p}^{2}}\left(\frac{P_{p}}{P}\right)^{2} \ln \left(\frac{1+\frac{B_{p}}{2 \Delta f_{p}}}{1-\frac{B_{p}}{2 \Delta f_{p}}}\right)\right] \int_{0}^{\infty}|\mathcal{K}(v)|^{2} d v$

where the sum is extended to all interfering pumps $p$ (or lightpaths) distinct from the reference lightpath with index $0, B_{p}$ is the p-th pump optical bandwidth, $\Delta f_{p}=$ $f_{p}-f_{0}$ is the carrier frequency offset of pump $p$ from reference channel $0, P_{p}$ is the p-th pump power ( $P$ is the power of the reference channel). The above formula can handle point-to-point WDM transmissions with flexible spectrum and format allocation, similarly to [11].

In classical coherent GN model treatments [4], [5], [10] that lead to eq. (11), all WDM channels enter at the same node $\mathrm{S}$ and exit at the same node $\mathrm{D}$, hence the kernel due to the pumps is the same as the kernel due to the reference channel (8). However, we now allow lightpath $p$ to enter at the generic coordinate $z_{i p}$ and exit at $z_{o p}$, with ${ }^{4} 0 \leq z_{i p}<z_{o p} \leq L$. The dual-polarization XCI NLI coefficient can now be approximated as:

$a_{X C I} \cong \frac{16}{27} \frac{B_{r x}}{B_{0}}\left[\sum_{p \neq 0} \frac{4}{B_{p}^{2}}\left(\frac{P_{p}}{P}\right)^{2} \ln \left(\frac{1+\frac{B_{p}}{2 \Delta f_{p}}}{1-\frac{B_{p}}{2 \Delta f_{p}}}\right) \int_{0}^{\infty}\left|\mathcal{K}_{p}(v)\right|^{2} d v\right]$

where

$$
\mathcal{K}_{p}(v)=\int_{z_{i p}}^{z_{o p}} \gamma_{p}(s) \mathcal{G}(s) \mathrm{e}^{-j \frac{(2 \pi)^{2} v}{2} C(s)} d s
$$

is the kernel due to pump $p$. The correctness of this formula is easily understood for links with identical gain $\mathcal{G}(s)$ on each span from the "classical" case where signal and pumps enter and exit at the same coordinates [10], by simply setting the nonlinear coefficient $\gamma_{p}(s)$ to zero in correspondence to segments of the signal path where the pump is absent. Now, for DU links with identical spans we exactly have [10]:

$$
\int_{0}^{\infty}\left|\mathcal{K}_{p}(v)\right|^{2} d v=N_{p} \mathcal{I}_{1}
$$

\footnotetext{
${ }^{4}$ If an interferer enters before $z=0$ we set $z_{i p}=0$. If it exits after $z=L$ we set $z_{o p}=L$.
}

where $\mathcal{I}_{1}=\int_{0}^{\infty}\left|\mathcal{K}_{\text {single }}(v)\right|^{2} d v$, and $\mathcal{K}_{\text {single }}$ is the single-span kernel, while the "interference length" $N_{p}$ is the number of spans from $z_{i p}$ to $z_{o p}$. Equation (14) implies that the XCI contributions of the various spans are completely uncorrelated in DU links (even within the coherent GN model), since the XCI NLI variance $a_{X C I} P^{3}$ scales linearly with $N_{p}$ [4], [10].

Also, note that what matters is just the interference length $N_{p}$ on wavelength $\lambda_{p}$ and not the exact entry/exit coordinates of the interfering lightpaths, nor how many lightpaths are active on $\lambda_{p}$ along the S-D path. Note that this fact holds for XCI within the framework of the coherent GN model for DU links, while it is automatically implied by the incoherent GN model [3], [11], [15, eq (2)]. For DU networks with identical spans and number of spans per hop $\left[\mathcal{S}_{1}, \ldots, \mathcal{S}_{H}\right]$ we can thus write $N_{p}=\sum_{k=1}^{H} \mathcal{S}_{k} I_{p k}$, where $I_{p k}=1$ if a lightpath is present on $\lambda_{p}$ at hop $k$ and 0 otherwise. Thus for DU networks we have from (12):

$$
a_{X C I} \cong \sum_{p \neq 0} \mathcal{C}_{p} \sum_{k=1}^{H} \mathcal{S}_{k} I_{p k}
$$

where

$$
\mathcal{C}_{p} \triangleq \frac{16}{27} \frac{B_{r x}}{B_{0}} \mathcal{I}_{1} \frac{4}{B_{p}^{2}}\left(\frac{P_{p}}{P}\right)^{2} \ln \left(\frac{1+\frac{B_{p}}{2 \Delta f_{p}}}{1-\frac{B_{p}}{2 \Delta f_{p}}}\right) .
$$

In the numerical examples in Section III we always used for all pumps $P_{p}=P, B_{p}=B_{0}$, and $\Delta f_{p}=$ $|p| \Delta f$, with $\Delta f$ the minimum channel spacing; also, $\mathcal{S}$ was equal at all hops.

We next assume the RVs $\left\{I_{p k}\right\}$ are independent for all $k$ and $p$, with mean $E\left[I_{p k}\right]=u_{k}$ equal for all wavelengths at hop $k$. Define the mean $\eta_{a} \triangleq E\left[a_{X C I}\right]$ and the variance $\sigma_{a}^{2} \triangleq \operatorname{Var}\left[a_{X C I}\right]$. By the independence assumption of the RVs $\left\{I_{p k}\right\}$, it is easy to get mean and variance:

$$
\begin{aligned}
\eta_{a} & =\left(\sum_{p \neq 0} \mathcal{C}_{p}\right) \sum_{k=1}^{H} \mathcal{S}_{k} u_{k} \\
\sigma_{a}^{2} & =\left(\sum_{p \neq 0} \mathcal{C}_{p}^{2}\right) \sum_{k=1}^{H} \mathcal{S}_{k}^{2}\left(1-u_{k}\right) u_{k}
\end{aligned}
$$

where we used the fact that $\operatorname{Var}\left[\mathcal{S}_{k} I_{p k}\right]=\mathcal{S}_{k}^{2} u_{k}\left(1-u_{k}\right)$. More sophisticated traffic models that include hop-byhop correlations can be accommodated.

Using (1), the SNR blocking event can be calculated as $\left\{S N R<S_{0}\right\}=\left\{a_{X C I}>\Theta\right\}$, where the threshold value $\Theta$ for $a_{X C I}$ above which an SB event occurs is:

$$
\Theta\left(P, N_{s}\right)=\frac{1}{S_{0} P^{2}}-\frac{\beta\left(N_{s}+H\right)}{P^{3}}-a_{S C I}\left(N_{s}\right)
$$


and depends both on power $P$ and on span number $N_{s}$. The SB probability $P_{S B}=\operatorname{Pr}\left\{S N R<S_{0}\right\}$ can thus be computed as

$$
P_{S B}=1-F_{a}(\Theta)
$$

where $F_{a}(x) \triangleq \operatorname{Pr}\left\{a_{X C I} \leq x\right\}$ is the cumulative distribution function (CDF) of the RV $a_{X C I}$.

Since the number of WDM wavelengths is normally large, it is clear from (15) that the RV $a_{X C I}$ will be the sum of many independent random variables without largely dominant terms, and by invoking the central limit theorem we may approximate it as a Gaussian RV, so that the SB probability can be approximated as

$$
1-F_{a}(\Theta) \cong \mathcal{Q}\left(\frac{\Theta\left(P, N_{s}\right)-\eta_{a}\left(\mathbf{u}, N_{s}\right)}{\sigma_{a}\left(\mathbf{u}, N_{s}\right)}\right)
$$

where $\mathcal{Q}(x) \triangleq \int_{x}^{\infty} \frac{1}{\sqrt{2 \pi}} \exp \left\{-u^{2} / 2\right\} d u$. $P_{S B}$ is thus the area below the Gaussian bell centered at $\eta_{a}$ with variance $\sigma_{a}^{2}$, from $\Theta$ to $\infty$. The threshold is load-independent. Hence as we increase the load $\mathbf{u}$ the bell shifts towards the threshold (i.e. $\eta_{a}$ increases). It broadens $\left(\sigma_{a}\right.$ increases) up to $u=0.5$ when variance reaches its maximum; at $u>0.5$ variance starts decreasing back to zero (reached at $u=1$ ).

The SB probability formula (19) and equations (16)(17) with equal pump powers $P_{p}=P$ and pump bandwidth $B_{p}=B_{0}$, and uniform channel spacing $\Delta f_{p}=|p| \frac{R}{\eta}$ were used in all numerical computations of (2) in Sec. III.

The SB contours at level $\mathcal{P}_{S B}$ shown in Fig. 1 were obtained by setting from (20):

$$
\Theta=F_{a}^{-1}\left(1-\mathcal{P}_{S B}\right) \cong \mathcal{Q}^{-1}\left(\mathcal{P}_{S B}\right) \sigma_{a}+\eta_{a}
$$

where $F_{a}^{-1}$ is the inverse CDF. Using (18), the above yields the following cubic equation in $P$ :

$$
P^{3}\left[a_{S C I}+F_{a}^{-1}\left(1-\mathcal{P}_{S B}\right)\right]-\frac{P}{S_{0}}+\beta\left(N_{s}+H\right)=0 .
$$

Its explicit Cardan's solutions (upper branch $P_{M}$ and lower branch $P_{m}$ ) are found as [9]:

$$
\begin{aligned}
& P_{M}=3 S_{0} \hat{N}_{A} \cos \left(\frac{\operatorname{acos}\left(-\beta\left(N_{s}+H\right) / \hat{N}_{A}\right)}{3}\right) \\
& P_{m}=3 S_{0} \hat{N}_{A} \cos \left(\frac{2 \pi-\operatorname{acos}\left(-\beta\left(N_{s}+H\right) / \hat{N}_{A}\right)}{3}\right)
\end{aligned}
$$

where

$$
\hat{N}_{A}=\frac{2}{\left(3 S_{0}\right)^{3 / 2} \sqrt{a_{S C I}\left(N_{s}\right)+F_{a}^{-1}\left(1-\mathcal{P}_{S B}\right)}}
$$

can be interpreted as the limit ASE power at which the two contour branches merge (when the acos(.) argument equals -1 we get $P_{M}=P_{m}$ from (22)-(23)). Hence the maximum number of spans $N_{0}$ at SB probability $\mathcal{P}_{S B}$ can be obtained by solving the implicit equation

$\beta\left(N_{0}(u)+H\right)=\frac{2}{\left(3 S_{0}\right)^{\frac{3}{2}} \sqrt{a_{S C I}\left(N_{0}\right)+F_{a}^{-1}\left(1-\mathcal{P}_{S B}, u, N_{0}\right)}}$

where we stressed in the notation that the threshold $\Theta=$ $F_{a}^{-1}\left(1-\mathcal{P}_{S B}\right)$ is also a function of both load $u$ and maximum number of spans $N_{0}$. The corresponding maxreach power is from (22)

$$
P_{0}(u)=3 S_{0} \beta\left(N_{0}+H\right) \cos \left(\frac{\pi}{3}\right)=\frac{3}{2} S_{0} \beta\left(N_{0}+H\right) .
$$

This is the key power value to be used by the loadaware RWA algorithm.

Note that at $\mathcal{P}_{S B}=1 / 2$ we have $\mathcal{Q}^{-1}\left(\mathcal{P}_{S B}\right)=0$, hence from (21): $\Theta=\eta_{a} \equiv E\left[a_{X C I}\right]$, and thus $a_{S C I}+$ $\Theta=E\left[a_{S C I}+a_{X C I}\right] \equiv E\left[a_{N L}\right]$. Thus the (22)-(23) contour at $\mathcal{P}_{S B}=1 / 2$ coincides with the contour of the SNR (3) calculated with the expected value $E\left[a_{N L}\right]$ of $a_{N L}$. This is readily seen by the SNR contour derivation in [9].

\section{COnCLusions}

For coherent transmissions in dispersionuncompensated flexible optical networks without all-optical wavelength conversion, we introduced a simple on-off interfering WDM traffic model that summarizes the impact of network traffic into a unique parameter, the average wavelength load $u$. We derived the statistics of the stochastic received SNR and thus the SNR blocking probability. From it, we derived analytical expressions of the load-dependent maximum reach $N_{0}(u)$ and its corresponding optimal power $P_{0}(u)$ within the framework of the coherent GN model. A novel load-aware RWA algorithm could then use the load-dependent reach $N_{0}(u)$ instead of the commonly used full-load reach $N_{0}(1)$. For such a novel load-aware RWA we quantified the possible savings in opto-electronic regenerations for two sample optical network topologies at the first wavelength blocking. For 28Gbaud WDM DP-QPSK signals at $80 \%$ bandwidth efficiency over $N_{s} \times 100 \mathrm{~km}$ SMF DU transmissions, regenerations savings of more than $20 \%$ and $38 \%$ are obtained in the US and EU networks, respectively, at the load corresponding to the first wavelength blocking. When single-channel nonlinearity is removed by an ideal DBP, the savings increase to more than $50 \%$ in the US and $60 \%$ in the EU network, respectively.

\section{APPENDIX 1}

Consider the generic contour (3), such as the red, blue, and dark green contours in Fig. 1. Let $N_{0}$ be 
the maximum number of reachable spans and $P_{0}$ the corresponding power. Then the point $\left(P_{0}, N_{0}\right)$ is the top of the bell curve (SNR versus power P) at distance $N_{0}$ when the top SNR value is $S_{0}$ [9]. But at the top of the bell curve, the ASE power $N_{A}=\beta\left(N_{0}+H\right)$ is twice the NLI power [7], hence the SNR is $S_{0}=$ $\frac{P_{0}}{N_{A}+N_{N L I}}=\frac{P_{0}}{\frac{3}{2} \beta\left(N_{0}+H\right)}$ i.e., $P_{0}=\frac{3}{2} S_{0} \beta\left(N_{0}+H\right)$ which is a straight line in the $(P, N)$ plane, shifted by $\left(\frac{3}{2}\right)^{d B} \cong 1.76 \mathrm{~dB}$ above the lower linear asymptote $P_{0}=S_{0} \beta\left(N_{0}+H\right)$. Such a line is the locus described by the coordinates of maximum reach as the NLI power (hence ASE, which has twice that power) is swept at constant $\beta$ (i.e., constant amplifiers noise figure $F$ ).

\section{REFERENCES}

[1] O. Rival, G. Villares, and A. Morea, "Impact of inter-channel nonlinearities on the planning of $25-100 \mathrm{~Gb} / \mathrm{s}$ elastic optical networks," J. Lightw. Technol., vol. 29, pp. 1326-1334, May 2011.

[2] E. Palkopoulou, G. Bosco, A. Carena, D. Klonidis, P. Poggiolini, and I. Tomkos, "Nyquist-WDM-Based Flexible Optical Networks: Exploring Physical Layer Design Parameters," J. Lightw. Technol., vol. 31, no. 14, pp. 2332-2339, Jul. 2013.

[3] P. Poggiolini, G. Bosco, A. Carena, R. Cigliutti, V. Curri, F. Forghieri, R. Pastorelli, and S. Piciaccia, "The LOGON Strategy for Low-Complexity Control Plane Implementation in NewGeneration Flexible Networks," Proc. OFC 2013, OW1H.3.

[4] P. Poggiolini, "The GN model of non-Linear propagation in uncompensated coherent optical systems," J. Lightwave Technol., vol. 30, pp. 3857-3879, Dec. 2012.

[5] P. Johannisson and M. Karlsson, "Perturbation analysis of nonlinear propagation in a strongly dispersive optical communication system," J. Lightw. Technol., vol. 31, no. 8, pp. 1273-1282, 2013.

[6] A. Bononi and P. Serena, "An alternative derivation of Johannisson's regular perturbation model," arXiv:1207.4729v1 [physics.optics], 2012.

[7] E. Grellier and A. Bononi, "Quality parameter for coherent transmissions with Gaussian-distributed nonlinear noise," OSA Opt. Express, vol. 19, no. 13, pp. 12781-12788, Jun. 2011.

[8] P. Poggiolini, A. Carena, V. Curri, G. Bosco, F. Forghieri, ”Analytical Modeling of Non-Linear Propagation in Uncompensated Optical Transmission Links," IEEE Photon. Technol. Lett., vol. 23, no. 11, pp. 742-744, Jun. 2011.

[9] A. Bononi, N. Rossi, and P. Serena, "On the nonlinear threshold versus distance in long-haul highly-dispersive coherent systems," OSA Opt. Express, vol. 20, no. 26, pp. B204-B216, Dec. 2012.

[10] A. Bononi, O. Beucher, and P. Serena, "Single- and crosschannel nonlinear interference in the Gaussian Noise model with rectangular spectra," OSA Opt. Express, vol. 21, no. 26, pp. 32254-32268, Dec. 2013.

[11] P. Johannisson and E. Agrell, "Modeling of Nonlinear Signal Distortion in Fiber-Optical Networks," arXiv:1309.4000v1 [physics.optics], Sep. 2013.

[12] A. Bononi, N. Rossi, P. Serena, "Performance Dependence on Channel symbol rate of Coherent Single-carrier WDM Systems," in Proc. ECOC'13, paper Th.1.D.5.

[13] A. Morea, N. Brogard, F. Leplingard, J.-C. Antona, T. Zami, B. Lavigne, and D. Bayart, "QoT function and $\mathrm{A}^{*}$ routing: an optimized combination for connection search in translucent networks," J. Opt. Netw., vol. 7, pp. 42-61. Jan. 2008.

[14] P. Péloso, D. Penninckx, M. Prunaire, and L. Noirie, "Optical transparency of a heterogeneous pan-European network," J. Lightw. Technol., vol. 22, pp. 242-248, Jan. 2004.

[15] D. J. Ives, A. Lord, P. Wright, and S. J. Savory, "Quantifying the Impact of Non-linear Impairments on Blocking Load in Elastic Optical Networks," in Proc. OFC'14, paper W2A.55. 